\title{
The role of delayed laparoscopic suction for intra-abdominal collection or abscess post appendectomy in paediatric patients: case series and review of literature
}

\author{
Dayang Anita Abdul Aziz¹, Surita Said¹, Marjmin Osman¹, Felicia Lim², Mahmud Mohd Nor ${ }^{1}$, Faizah Mohd Zaki \\ Zarina Abdul Latiff ${ }^{4}$
}

${ }^{I}$ Paediatric Surgery Unit, Department of Surgery, Universiti Kebangsaan Malaysia Medical Centre (UKMMC), Jalan Yaacob Latiff, Cheras, Kuala Lumpur 56000, Malaysia.

${ }^{2}$ Department of Anaesthesia, Universiti Kebangsaan Malaysia Medical Centre (UKMMC), Jalan Yaacob Latiff, Cheras, Kuala Lumpur 56000, Malaysia. ${ }^{3}$ Department of Radiology, Universiti Kebangsaan Malaysia Medical Centre (UKMMC), Jalan Yaacob Latiff, Cheras, Kuala Lumpur 56000, Malaysia. ${ }^{4}$ Department of Paediatrics, Universiti Kebangsaan Malaysia Medical Centre (UKMMC), Jalan Yaacob Latiff, Cheras, Kuala Lumpur 56000, Malaysia.

Correspondence to: Prof. Dayang Anita Abdul Aziz, Paediatric Surgery Unit, Department of Surgery, Universiti Kebangsaan Malaysia Medical Centre (UKMMC), Jalan Yaacob Latiff, Cheras, Kuala Lumpur 56000, Malaysia. E-mail: dayanganita@yahoo.co.uk

How to cite this article: Aziz DAA, Said S, Osman M, Lim F, Mohd Nor M, Mohd Zaki F, Latiff ZA. The role of delayed laparoscopic suction for intra-abdominal collection or abscess post appendectomy in paediatric patients: case series and review of literature. Mini-invasive Surg 2017;1:143-7.

Article history:

Received: 8 Jun 2017

Accepted: 15 Aug 2017

Published: 30 Sep 2017

Key words:

Intraabdominal abscess,

appendicitis,

laparoscopic,

paediatrics

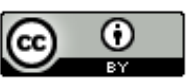

This is an open access article licensed under the terms of Creative Commons Attribution 4.0 International License (https://creativecommons.org/licenses/by/4.0/), which permits unrestricted use, distribution, and reproduction in any medium, as long as the original author is credited and the new creations are licensed under the identical terms.

For reprints contact: service@oaepublish.com

\begin{abstract}
A BSTRACT
Aim: Intra-abdominal collection or abscess (IAA) is a dreaded complication post open or laparoscopic appendectomy for perforated appendicitis. There have been many discussions on the role of laparoscopic irrigation during laparoscopic appendectomy for perforated appendix but not its role for patients who subsequently developed IAA post-surgery. Methods: All patients who developed clinical symptoms and radiological evidence of IAA of more than $5 \mathrm{~cm} \times 5 \mathrm{~cm}$ post appendectomy from January 2014 to May 2016 were subjected to delayed laparoscopic suction (DLS) of the IAA. Days to resolution of fever and improvement of symptoms post the DLS were recorded. Complications during DLS like bowel injury, bleeding and conversion to open surgery were documented and analysed. Patients were followed up for 1 month to a year to look for potential adhesive intestinal obstruction. Results: Seven patients who met the criteria of large IAA were subjected to DLS at post-operative day 3 to day 5 post appendectomy. Six of the cases were post laparoscopic appendectomy and one case was post open appendectomy from another institution. Ports were inserted via the same sites as used during the first surgery. Turbid intraperitoneal fluid and abscesses were laparoscopically sucked without irrigation. There was no bowel injury, bleeding or conversion in any of the cases. All patients were afebrile within $24 \mathrm{~h}$ post procedure and their associated symptoms improved significantly. All patients were discharged within three days of DLS and have not returned with adhesive obstruction. Conclusion: Early recognition of IAA is important and early attempt at DLS resulted in better outcome of patients and lesser hospital stay. DLS is a safe and feasible technique.
\end{abstract}




\section{INTRODUCTION}

Laparoscopic appendectomy (LA) for perforated appendicitis had shown favorable outcomes in terms of length of hospital stay, antibiotic usage, return of oral intake and rate of wound infection in comparison with open appendectomy (OA). With regards to infection rate, Lin et al. ${ }^{[1]}$ in 2006 showed that the rate of wound infection was lower than the OA group i.e. $15.2 \%$ vs. $30.7 \%$. However, there was no mention of the more dreaded complication of intra-abdominal abscess (IAA) especially after LA for perforated appendicitis. This issue was later addressed in subsequent literature and it became a major concern when deciding to perform LA for perforated appendicitis for many surgeons. The European guideline recommends thorough peritoneal lavage (6-8 $L$ of warm saline) and aspiration to minimize the IAA rate in complicated appendicitis. However, this practice was controversial as it was postulated that lavage itself might help to spread the infectious materials. ${ }^{[2-4]}$

There are many published articles on the role of $L A$ and lavage and $O A$ and peritoneal washout in the formation of IAA, however there has not been any discussion on the management of these patients with IAA postsurgery except for placement of drains and antibiotics in some series. ${ }^{[5-9]}$ The aim of this study is to document feasibility and effectiveness of delayed laparoscopic suction (DLS) at tackling IAA. The hypothesis is that DLS is a feasible and effective technique for treatment of IAA post perforated appendectomy in children.

\section{METHODS}

This study was based on a comprehensive review of audit on paediatric patients who underwent laparoscopic appendectomy at our institution for acute and perforated appendicitis for the recent three and a half years; we looked at the occurrence of IAA and the management strategy to resolve this problem i.e. percutaneous drainage or DLS. All patients with acute or perforated appendicitis at our institution would undergo laparoscopic appendectomy unless there was presence of distended abdomen from a dilated bowel i.e. suggestive of intestinal obstruction secondary to the pathological appendix. Suggestion of intestinal obstruction would be evident clinically and supported radiologically (AXR and/or ultrasound abdomen). We documented the type of appendicitis (suppurative or perforated), whether irrigation and suction was done intraoperatively, days of persistent and cessation of fever, presence of abdominal pain and poor appetite and disappearance of these symptoms, ultrasound findings of the measurement of the IAA, days of administration of standard antibiotics (and additional antibiotics if any) and days to discharge. We also documented clinical evaluation of these patients during follow-up, specifically looking for symptoms to suggest adhesive intestinal obstruction. We also included in this series, cases referred to our centre for management of IAA. Parental consent was taken prior to DLS procedure.

The laparoscopic approach to appendectomy was the 3-port technique using $11 \mathrm{~mm}$ Hasson trocar for camera insertion and 2-6 mm working ports. The antibiotics of choice were intravenous second generation cephalosporin group and metronidazole. The surgical technique was standardised for all patients. All appendiceal stumps were ligated using loop polypropylene suture. All perforated cases would have suction and irrigation with unspecified amount of warm saline till the effluent was clear. Patients who have persistent fever at day 3 of post-surgery with or without symptoms of abdominal distension, pain or poor appetite would be subjected to ultrasound assessment to look for presence of IAA, its complexity and size. Intraabdominal abscess of less than $5 \mathrm{~cm} \times 5 \mathrm{~cm}$ were treated conservatively by adding intravenous gentamycin (aminoglycoside). For cases with IAA of more than $5 \mathrm{~cm} \times 5 \mathrm{~cm}$, we documented the procedures chosen to manage the IAA i.e. either percutaneous drainage or DLS and the clinical progress based on factors mentioned earlier.

\section{RESULTS}

Out of the 49 cases of LA at our institution, 20 cases were for perforated appendicitis and 29 were for suppurative appendicitis. None of the cases underwent conversion to open surgery. Intraoperatively, all cases with perforated appendicitis had laparoscopic peritoneal lavage with unspecified amount of warm saline and suction. Out of 20 patients, 9 developed IAA.

All 9 patients with suspected IAA were febrile at postoperative day 3 with temperature of more than $38^{\circ} \mathrm{C}$. They were subjected to ultrasound abdomen for confirmation of IAA. Ultrasound showed 8 patients had IAA larger than $5 \mathrm{~cm} \times 5 \mathrm{~cm}$ at the right iliac fossa and in the pelvis region. Two patients underwent percutaneous drainage under ultrasound guidance and a pigtail catheter insertion, the tip of the catheter was placed in the pelvic cavity to drain the residual IAA into a sterile bag. The aspirated pus was sent for culture and sensitivity and the bacterial involved was confirmed to be Escherichia coli. In these 2 patients, intravenous antibiotic gentamycin was added. Both patients remained febrile till over a week post-surgery. 
They also complaint of pain at the site of the pigtailcatheter and difficulty to mobilise, having to carry the catheter and drainage bag all the time. However, their appetite improved after the percutaneous drainage. They were discharged at POD10 and POD14 respectively after repeat ultrasound showed complete resolution of IAA and removal of the pigtail-catheter. At follow up, 1 patient complaint of pain at the right iliac fossa with no symptoms to suggest adhesive obstruction. A repeat ultrasound was done for him which was normal. The second patient remained well at follow-up.

One patient with IAA of $3 \mathrm{~cm} \times 4 \mathrm{~cm}$ was treated conservatively; intravenous Gentamycin was added and she became afebrile $48 \mathrm{~h}$ later. This patient's appetite took longer time to resolve, however she did not complain of abdominal pain. She was subsequently discharged well at POD7. Repeat ultrasound was not done on her before discharge; at follow-up, she was also well.

Six other patients with IAA of more than $5 \mathrm{~cm} \times 5 \mathrm{~cm}$ on were subjected to DLS. All of them were febrile with temperature of $38{ }^{\circ} \mathrm{C}$ or more at POD3. Three patients had no abdominal pain and their appetite were normal. Three other patients have either one or more combination of symptoms e.g. fever, abdominal pain, poor appetite and refusal to mobilise [Table 1]. All 6 patients underwent DLS at POD3 to POD5 via the same port-sites used during first surgery. No additional antibiotics was given. During DLS, intraoperatively, only laparoscopic suction was carried out without irrigation. Post procedure, fever resolved within $24 \mathrm{~h}$ of DLS in all 6 patients. Patients who had complaints had complete resolution of their symptoms. They were discharged as early as second to third day post-DLS. The 7th patient included in this review was a patient who underwent open appendectomy for perforated appendicitis at another institution. The appendectomy scar was consistent with a standard Lantz incision about $5 \mathrm{~cm}$ in length. The patient was referred to us at POD3 after abdominal ultrasound revealed an $8 \mathrm{~cm} \times 5 \mathrm{~cm}$ collection in the pelvis; the patient also had persistent high-grade fever $\left(39^{\circ} \mathrm{C}\right)$. DLS was performed at POD4; the fever settled within $24 \mathrm{~h}$ and patient was discharged two days after DLS. All 7 patients who underwent DLS did not have any complications such as bowel injury, serosal tear or any difficulty in suctioning out the IAA during procedure.

Comparing the patients who underwent DLS and those who had percutaneous drainage, the DLS-group had early cessation of fever (a day after the procedure); they also went home earlier than the percutaneous group (at about 2-3 days post-DLS and total length of stay in the ward did not exceed more than a week). The percutaneous-drainage-group took longer time to become afebrile (fever was settled after more than a week); they also had additional pain and discomfort from the pigtail-insertion. They also stayed longer in the ward (10 and 14 days).

Follow-up was carried out at 1-month post-surgery, all DLS-grouped patients remained well clinically and backed to their normal selves. The histopathological examinations of their appendix confirmed perforated appendicitis. None of the patients had symptoms for adhesive obstruction.

\section{DISCUSSION}

A study published in 2014 by Taguchi et al. ${ }^{[5]}$ from Nagoya Red Cross Hospital, Japan, showed no significant difference in the incident of IAA formation between LA and $O A$ in treating complicated appendicitis i.e. 17 vs. 20. This single-centre randomized-controlled trial was conducted with the development of an infectious complications including IAA formation as primary outcome. In this study, the operating surgeon performed thorough peritoneal lavage using several liters of warm saline regardless of whether an abscess or peritonitis was present. This study showed the safety and feasibility of the usage of LA for complicated appendicitis. Most importantly

Table 1: Summary of clinical presentations and progress

\begin{tabular}{|c|c|c|c|c|c|c|c|c|}
\hline \multirow{2}{*}{$\begin{array}{l}\text { No. of } \\
\text { patients }\end{array}$} & \multicolumn{4}{|c|}{ Associated symptoms } & \multirow{2}{*}{$\begin{array}{l}\text { Day of performing } \\
\text { DLS from original } \\
\text { surgery }\end{array}$} & \multirow{2}{*}{$\begin{array}{l}\text { Time post-DLS } \\
\text { that fever settled }\end{array}$} & \multirow{2}{*}{$\begin{array}{c}\text { Day of } \\
\text { discharge } \\
\text { post-DLS }\end{array}$} & \multirow{2}{*}{$\begin{array}{l}\text { Total length of } \\
\text { stay in ward } \\
\text { (days) }\end{array}$} \\
\hline & $\begin{array}{l}\text { Persistent fever at POD3 } \\
\text { and temperature }\left({ }^{\circ} \mathrm{C}\right)\end{array}$ & $\begin{array}{l}\text { Abdominal } \\
\text { pain }\end{array}$ & $\begin{array}{l}\text { Poor } \\
\text { appetite }\end{array}$ & $\begin{array}{l}\text { Refusal to } \\
\text { mobilise }\end{array}$ & & & & \\
\hline 1 & Yes, 38.7 & Yes & No & Yes & POD3 & POD-DLS 1 & POD-DLS 3 & 6 \\
\hline 2 & Yes, 38.5 & Yes & No & No & POD4 & POD-DLS 1 & POD-DLS 3 & 7 \\
\hline 3 & Yes, 39.0 & No & Yes & Yes & POD4 & POD-DLS 1 & POD-DLS 3 & 7 \\
\hline 4 & Yes, 39.0 & Yes & Yes & Yes & POD3 & POD-DLS 1 & POD-DLS 3 & 6 \\
\hline 5 & Yes, 38.5 & No & No & Yes & POD5 & POD-DLS 1 & POD-DLS 2 & 7 \\
\hline 6 & Yes, 39.0 & No & No & Yes & POD4 & POD-DLS 1 & POD-DLS 2 & 6 \\
\hline 7 & Yes, 39.0 & Yes & Yes & Yes & POD4 & POD-DLS 1 & POD-DLS 2 & 6 \\
\hline
\end{tabular}

DLS: delayed laparoscopic suction; POD: post-operative day; POD-DLS: post-operative day- delayed laparoscopic suction 
it showed that it was not the type of approach (LA vs. $\mathrm{OA}$ ) that determined the rate of IAA formation post appendectomy, but some other factors that yet to be determined. In this study, all the patients with infected wound underwent open surgery to obtain optimal drainage followed by lavage with a water shower. Drain was then placed in most of the cases. It did prolong the healing time but was comparable in both groups..$^{\left[{ }^{5]}\right.}$

In 2015, Cho et al. ${ }^{[6]}$ conducted a study with the aim to identify the risk factors for IAA formation in patients receiving $L A ; 1,817$ patients were enrolled in this study. During the LA, when the surgical findings revealed generalized peritonitis or pus, or if faecolith were spilled during the procedure, the operative surgeon would routinely perform cleansing procedure using laparoscopic gauze and suction; no irrigation water was involved. However, if the routine cleansing procedure was incomplete, peritoneal irrigation using sterile isotonic saline with minimum volume of $200 \mathrm{~mL}$ would be carried out. Result showed that 27 patients (1.5\%) developed IAA formation; 21 of them had received peritoneal irrigation intra-operatively and this was the only factor that was statistically significant to raise the incident of IAA formation in this study. Not even the type of appendicitis (suppurative vs. complicated) or metabolic factor (diabetes vs. non-diabetes) or use of antibiotics could significantly contribute to the higher incidence of IAA formation. IAA was suspected when patient developed fever and abdominal pain post operatively. Computed tomography scan abdomen was performed to confirm the IAA formation. Among the 27 patients who developed IAA postoperatively, only 1 patient received a re-operation. However, the type of operation (laparoscopic or open) and procedure done was not explained in this paper. The remaining 26 cases of IAA, 12 of them underwent percutaneous drainage, and the rest received antibiotic treatment only, no mortality was reported. ${ }^{[6]}$

A comparative study done by Moore et al. ${ }^{[7]}$ published in 2011, documented a higher abscess rate when irrigation was used during appendectomy for perforated appendicitis including LA. In this study, the data of 176 patients who underwent appendectomy (39\% open and $61 \%$ laparoscopic) were reviewed retrospectively. More than $50 \%$ of patients in both groups received intra-operative irrigation. The amount of irrigation was not quantified in this study due to inconsistent amount of irrigation used between surgeons. Perforation was observed in $28 \%$ (50/176), of which $86 \%(43 / 50)$ of patients received intraoperative irrigation. Eleven patients $(9.6 \%)$ with irrigation developed postoperative abscess compared with $2(3.3 \%)$ patients without irrigation $(P=0.22)$. The result showed no decrease in postoperative intra-abdominal abscess with use of intraoperative irrigation. They concluded that routine use of intraoperative irrigation for appendectomies does not prevent intra-abdominal abscess formation. This paper did not mention specifically the subsequent management of postoperative IAA among their patients. ${ }^{[7]}$

A prospective randomised trial study conducted by St Peter et al. $^{\left[{ }^{[8]}\right.}$ in 2012, concluded that there is no advantage to irrigation of the peritoneal cavity over suction alone during laparoscopic appendectomy for perforated appendicitis; 220 patients with perforated appendicitis were enrolled in this study. They were randomised to irrigation (minimum of $500 \mathrm{~mL}$ of saline with no maximum limit), or to suction only group (no irrigation). There were 110 patients treated in each arm of this study. There were no differences in age, weight, body mass index percentile, gender distribution, duration of symptoms, presenting leukocyte count, or temperature between the 2 groups. All patients were managed with the same antibiotic regiment and protocol. The primary outcome variable was the development of a postoperative abdominal abscess. The result showed no difference in abscess rate, which was $19.1 \%$ with suction only group and $18.3 \%$ in irrigation group $(P=1.0)$. From these data, it's clearly demonstrated that the outcome in patients with perforated appendicitis will not be affected by the use of a moderate amount of irrigation during laparoscopic appendectomy. The authors did not mention regarding the management of postoperative IAA among their patients. ${ }^{[8]}$

Similar result was found in recent randomised, controlled equivalence trial done by Snow et al. ${ }^{\left[{ }^{9}\right]}$ that was published in 2016. Eighty-two patients with suppurative or perforated appendicitis were enrolled in this study; 41 patients were randomised to the suction only (SO) group and 40 patients to irrigation and suction (IS) group. Procedure was performed using standard laparoscopic technique. Primary end point was the rate of IAA formation. A median volume of $675 \mathrm{~mL}$ of irrigation was used in the IS group. Result showed equal number of patient who developed IAA in both groups (5\% in IS group and $4.9 \%$ in SO group). From the 4 patients with IAA, 3 required re-operation, in which 2 were laparoscopic (1 SO, 1 IS) and 1 by laparotomy (SO). Another patient (IS) was planned for percutaneous drainage but this was cancelled due to decreasing size of the collection. The rate of IAA in this study was lower than other studies, which had shown closer to $20 \%$. The reason being is most likely due to the case definition for enrollment. This study included suppurative appendicitis as their subjects while other studies focused more on perforated appendicitis. ${ }^{[9]}$

In our series, persistent fever was the most 
reliable symptom to suspect IAA or collection post appendectomy. Ultrasound was the only radiological tool needed for our patients to confirm the diagnosis of IAA compared to the other series. There was no IAA following appendectomy for suppurative appendicitis in our series. From our review, DLS was easy to do. It provided us with immediate cessation of fever for the patients and relieved of their symptoms (abdominal pain poor appetite and refusal to ambulate). Patients were able to mobilise faster post-DLS too, compared to those who had percutaneous drainage. Patients were also able to be discharged earlier than those who underwent percutaneous drainage. Technically, DLS did not require any additional port insertion and the technique of DLS was quite straight forward, the ease is probably because the abscesses were still early in their phase and less complicated. This technique was also feasible for patient who had undergone open appendectomy; provided the scar did not interfere with area for port insertion.

Following success of this series, we would like to propose a simple guideline on laparoscopic management of IAA post-appendectomy [Figure 1]. Intraabdominal abscess post appendectomy is not a complication that any centre would like to have in high numbers, as a result of the low incidence, we were not able to show statistical significance to compare results between DLS and percutaneous drainage of IAA. However, we were able to demonstrate how a surgeon would be able to resolve this complication quickly. There has not been any literature describing this technique. In conclusion, we believe DLS is a safe, fast and feasible technique to treat IAA. We recommend

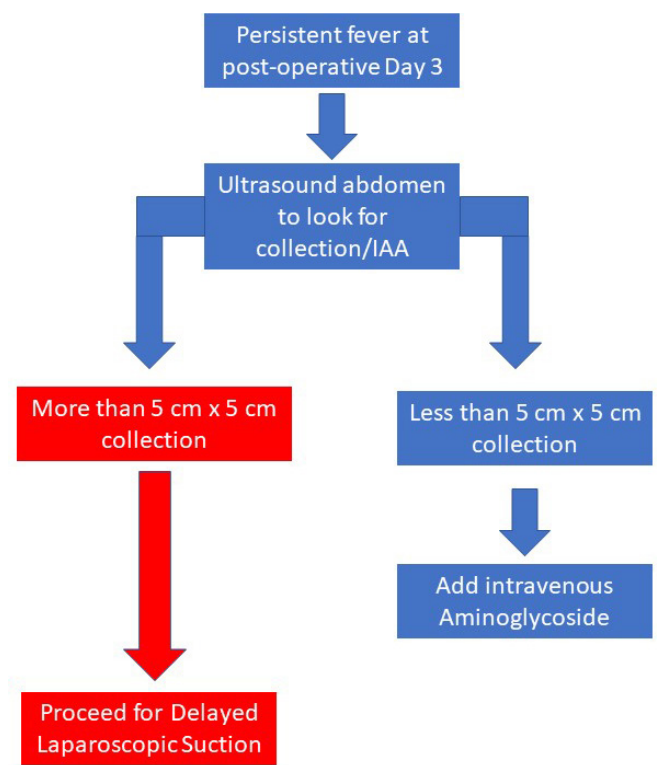

Figure 1: Pathway for management of intra-abdominal abscess (IAA) post-appendectomy this technique for all centres which advocate minimally invasive technique in children.

\section{DECLARATIONS}

\section{Authors' contributions}

Original idea and drafting of text: D.A.A. Aziz, Z.A. Latiff

Literature review and data collection: S. Said, F. Lim, M. Mohd Nor

Data analysis: M. Osman, F. Mohd Zaki

\section{Financial support and sponsorship}

None.

\section{Conflicts of interest}

There are no conflicts of interest.

\section{Patient consent}

Parental consent was obtained for all patients.

\section{Ethics approval}

Reviews based on our existing audit is waived for ethical approval.

\section{REFERENCES}

1. Lin HF, Wu JM, Tseng LM, Chen KH, Huang SH, Lai IR. Laparoscopic versus open appendectomy for perforated appendicitis. J Gastrointest Surg 2006;10:906-10.

2. Frazee RC, Bohannon WT. Laparoscopic appendectomy for complicated appendicitis. Arch Surg 1996;131:509-11; discussion 511-3.

3. Bonanni F, Reed J 3rd, Hartzell G, Trostle D, Boorse R, Gittleman M, Cole A. Laparoscopic versus conventional appendectomy. J Am Coll Surg 1994;179:273-8.

4. Krisher SL, Browne A, Dibbins A, Tkacz N, Curci M. Intra-abdominal abscess after laparoscopic appendectomy for perforated appendicitis. Arch Surg 2001;136:438-41.

5. Taguchi Y, Komatsu S, Sakamoto E, Norimizu S, Shingu Y, Hasegawa H. Laparoscopic versus open surgery for complicated appendicitis in adults: a randomized controlled trial. Surg Endosc 2016;30:1705-12.

6. Cho J, Park I, Lee D, Sung K, Baek J, Lee J. Risk factors for postoperative intra-abdominal abscess after laparoscopic appendectomy: analysis for consecutive 1,817 experiences. Dig Surg 2015;32:375-81

7. Moore CB, Smith RS, Herbertson R, Toevs C. Does use of intraoperative irrigation with open or laparoscopic appendectomy reduce post-operative intra-abdominal abscess? Am Surg 2011;77:78-80.

8. St Peter SD, Adibe OO, Iqbal CW, Fike FB, Sharp SW, Juang D, Lanning D, Murphy JP, Andrews WS, Sharp RJ, Snyder CL, Holcomb $\mathrm{GW}$, Ostlie DJ. Irrigation versus suction alone during laparoscopic appendectomy for perforated appendicitis: a prospective randomized trial. Ann Surg 2012;256:581-5.

9. Snow HA, Choi JM, Cheng MW, Chan ST. Irrigation versus suction alone during laparoscopic appendectomy; a randomized controlled equivalence trial. Int J Surg 2016;28:91-6. 